\title{
Solutions of non-homogeneous linear differential equations in the unit disc
}

\author{
by Ting-Bin CaO and Zhong-Shu Deng (Nanchang)
}

\section{Dedicated to Professor Zong-Xuan Chen on the occasion of his 60th birthday}

\begin{abstract}
The main purpose of this paper is to consider the analytic solutions of the non-homogeneous linear differential equation

$$
f^{(k)}+a_{k-1}(z) f^{(k-1)}+\cdots+a_{1}(z) f^{\prime}+a_{0}(z) f=F(z),
$$

where all coefficients $a_{0}, a_{1}, \ldots, a_{k-1}, F \not \equiv 0$ are analytic functions in the unit disc $\mathbb{D}=$ $\{z \in \mathbb{C}:|z|<1\}$. We obtain some results classifying the growth of finite iterated order solutions in terms of the coefficients with finite iterated type. The convergence exponents of zeros and fixed points of solutions are also investigated.
\end{abstract}

1. Introduction and main results. In this paper, we assume that the reader is familiar with the fundamental results and standard notations of Nevanlinna's value distribution theory of meromorphic functions on the complex plane $\mathbb{C}$ and in the unit disc $\mathbb{D}=\{z \in \mathbb{C}:|z|<1\}$ (see [9], [21]). First, the weighted Hardy space $H_{q}^{\infty}$ consists of those functions $f$, analytic in the unit disc, such that

$$
\sup _{0 \leq r<1} M(r, f)\left(1-r^{2}\right)^{q}<\infty,
$$

where $M(r, f)$ is the maximum modulus of $f$ on the circle of radius $r$ centered at the origin. If $g$ belongs to $H_{q}^{\infty}$ for some $q \geq 0$, then it is said to be an $H$-function. One can deduce that

$$
D_{M}(f):=\limsup _{r \rightarrow 1^{-}} \frac{\log ^{+} M(r, f)}{-\log (1-r)}=\inf \left\{q \geq 0: f \in H_{q}^{\infty}\right\},
$$

2010 Mathematics Subject Classification: Primary 34M10; Secondary 30D35.

Key words and phrases: differential equation, analytic function, iterated order, iterated convergence exponent of zeros, unit disc. 
where $\log ^{+} x=\max \{\log x, 0\}$. If $f$ is meromorphic in $\mathbb{D}$ satisfying

$$
D(f):=\limsup _{r \rightarrow 1^{-}} \frac{T(r, f)}{-\log (1-r)}<\infty \quad(\text { resp. }=\infty),
$$

then we say that $f$ is non-admissible (resp. admissible), where $T(r, f)$ is the Nevanlinna characteristic of $f$. If $f$ is analytic in $\mathbb{D}$, then $D_{M}(f)$ and $D(f)$ satisfy the inequality $D(f) \leq D_{M}(f)$. This meanss that every analytic admissible function in $\mathbb{D}$ is an $H$-function. Furthermore, there are analytic functions, such as $f=e^{1 /(1-z)}$ (see Remark 1.5 of [5]), satisfying $D(f)<D_{M}(f)$.

For $n \in \mathbb{N}$, the iterated $n$-order of a meromorphic function $f$ in $\mathbb{D}$ is defined by

$$
\sigma_{n}(f):=\limsup _{r \rightarrow 1^{-}} \frac{\log _{n}^{+} T(r, f)}{-\log (1-r)},
$$

where $\log _{1}^{+} x=\log ^{+} x$ and $\log _{n+1}^{+}=\log ^{+} \log _{n}^{+} x$. If $f$ is analytic in $\mathbb{D}$, we also define

$$
\sigma_{M, n}(f):=\limsup _{r \rightarrow 1^{-}} \frac{\log _{n+1}^{+} M(r, f)}{-\log (1-r)} .
$$

If $f$ is analytic in $\mathbb{D}$, it is well known that $\sigma_{M, 1}(f)$ and $\sigma_{1}(f)$ satisfy the inequalities

$$
\sigma_{1}(f) \leq \sigma_{M, 1}(f) \leq \sigma_{1}(f)+1,
$$

which are best possible in the sense that there are analytic functions $g$ and $h$ such that $\sigma_{M, 1}(g)=\sigma_{1}(g)$ and $\sigma_{M, 1}(h)=\sigma_{1}(h)+1$ (see [8]). However, it follows from Proposition 2.2.2 of [14] that $\sigma_{M, n}(f)=\sigma_{n}(f)$ for $n \geq 2$.

For $n \in \mathbb{N}$, the iterated $n$-convergence exponent of the sequence of zeros in $\mathbb{D}$ of a meromorphic function $f$ in $\mathbb{D}$ is defined by

$$
\lambda_{n}(f):=\limsup _{r \rightarrow 1^{-}} \frac{\log _{n}^{+} N(r, 1 / f)}{-\log (1-r)}
$$

and $\bar{\lambda}_{n}(f)$, the iterated $n$-convergence exponent of the sequence of distinct zeros in $\mathbb{D}$ of $f$ is defined by

$$
\bar{\lambda}_{n}(f):=\limsup _{r \rightarrow 1^{-}} \frac{\log _{n}^{+} \bar{N}(r, 1 / f)}{-\log (1-r)} .
$$

Recently, there has been an increasing interest in studying the growth of analytic solutions of

$$
f^{(k)}+a_{k-1}(z) f^{(k-1)}+\cdots+a_{1}(z) f^{\prime}+a_{0}(z) f=0
$$

in the unit disc $\mathbb{D}$. For finite 1-order solutions, see [3, 8, 12, 10, 13, 7], and for finite $n$-order solutions, see [5, 11]. In a very recent paper [4, the convergence exponent of the sequence of zeros of solutions of linear differential equations in $\mathbb{D}$ has been considered. 
The main purpose of this paper is to consider the analytic solutions of the non-homogeneous linear differential equation, corresponding to (1),

$$
f^{(k)}+a_{k-1}(z) f^{(k-1)}+\cdots+a_{1}(z) f^{\prime}+a_{0}(z) f=F(z),
$$

where all coefficients $a_{0}, a_{1}, \ldots, a_{k-1}, F \not \equiv 0$ are analytic functions in $\mathbb{D}$. The first result classifies the growth of finite iterated 1-order solutions of (2) in terms of the growth of the coefficients.

TheOREM 1.1. Let $a_{j}(j=0,1, \ldots, k-1)$ be $H$-functions and assume that $F \not \equiv 0$ and

$$
\sigma_{M, 1}(F) \leq \max \left\{0, \max _{j=0, \ldots, k-1} \frac{D_{M}\left(a_{j}\right)}{k-j}-1\right\} .
$$

In particular, $F$ may be an $H$-function. Then all solutions $f$ of (2) satisfy

$$
\sigma_{1}(F) \leq \sigma_{1}(f) \leq \sigma_{M, 1}(f) \leq \max \left\{0, \max _{j=0, \ldots, k-1} \frac{D_{M}\left(a_{j}\right)}{k-j}-1\right\} .
$$

Moreover, if either $F$ is an $H$-function or $\sigma_{1}(F)<\sigma_{1}(f)$, then $\lambda_{1}(f)=$ $\bar{\lambda}_{1}(f)=\sigma_{1}(f)$.

Next, we obtain the following results classifying the growth of finite iterated $n$-order solutions of (2) in terms of the iterated $n$-type of coefficients. For $n \in \mathbb{N}$, the iterated $n$-type of an analytic function $f$ in $\mathbb{D}$ with $0<$ $\sigma_{M, n}(f)<\infty$ is defined by

$$
\tau_{M, n}(f):=\limsup _{r \rightarrow 1}(1-r)^{\sigma_{M, n}(f)} \log _{n}^{+} M(r, f) .
$$

THEOREM 1.2. Let $n \in \mathbb{N}$, and assume that the coefficients $a_{0}, \ldots, a_{k-1}$ and $F \not \equiv 0$ are analytic in $\mathbb{D}$ with $\sigma_{M, n}\left(a_{j}\right) \leq \sigma_{M, n}\left(a_{0}\right)$ for all $j=1, \ldots, k-1$, and

$$
\sum_{\sigma_{M, n}\left(a_{j}\right)=\sigma_{M, n}\left(a_{0}\right)} \tau_{M, n}\left(a_{j}\right)<\tau_{M, n}\left(a_{0}\right) .
$$

(i) If $\sigma_{M, n+1}(F)>\sigma_{M, n}\left(a_{0}\right)$, then all solutions $f$ of (2) satisfy $\sigma_{M, n+1}(f)=\sigma_{M, n+1}(F)$.

(ii) If $\sigma_{M, n+1}(F)<\sigma_{M, n}\left(a_{0}\right)$, then all solutions $f$ of (2) satisfy $\sigma_{M, n+1}(f)=\lambda_{n+1}(f)=\bar{\lambda}_{n+1}(f)=\sigma_{M, n}\left(a_{0}\right)$, with at most one exception $f_{0}$ satisfying $\sigma_{M, n+1}\left(f_{0}\right)<\sigma_{M, n}\left(a_{0}\right)$.

If $\sigma_{M, n}\left(a_{j}\right)<\sigma_{M, n}\left(a_{0}\right)$ for all $j=1, \ldots, k-1$, then the sum of (3) is empty, and Theorem 1.2 reduces to the following result.

Corollary 1.1. Let $n \in \mathbb{N}$, and assume that the coefficients $a_{0}, \ldots, a_{k-1}$ and $F \not \equiv 0$ are analytic in $\mathbb{D}$ and $\sigma_{M, n}\left(a_{j}\right)<\sigma_{M, n}\left(a_{0}\right)$ for all $j=1, \ldots, k-1$. Then both (i) and (ii) of Theorem 1.2 are true.

If the condition $\sigma_{M, n}\left(a_{j}\right)<\sigma_{M, n}\left(a_{0}\right)$ is replaced by $\sigma_{n}\left(a_{j}\right)<\sigma_{n}\left(a_{0}\right)$, then we have the following result. 
TheOREM 1.3. Let $n \in \mathbb{N}$, and assume that the coefficients $a_{0}, \ldots, a_{k-1}$ and $F \not \equiv 0$ are analytic in $\mathbb{D}$ and $\sigma_{n}\left(a_{j}\right)<\sigma_{n}\left(a_{0}\right)$ for all $j=1, \ldots, k-1$. Let $\alpha_{M}:=\max \left\{\sigma_{M, n}\left(a_{j}\right): j=0, \ldots, k-1\right\}$.

(i) If $\sigma_{M, n+1}(F)>\alpha_{M}$, then all solutions $f$ of (2) satisfy $\sigma_{M, n+1}(f)=$ $\sigma_{M, n+1}(F)$.

(ii) If $\sigma_{M, n+1}(F)<\alpha_{M}$, then all solutions $f$ of (2) satisfy $\sigma_{n}\left(a_{0}\right) \leq$ $\sigma_{M, n+1}(f) \leq \alpha_{M}$, with at most one exception $f_{0}$ satisfying $\sigma_{M, n+1}\left(f_{0}\right)$ $<\sigma_{n}\left(a_{0}\right)$.

(iii) If $\sigma_{M, n+1}(F)<\sigma_{n}\left(a_{0}\right)$, then all solutions $f$ of (2) satisfy $\alpha_{M} \geq$ $\sigma_{M, n+1}(f)=\lambda_{n+1}(f)=\bar{\lambda}_{n+1}(f) \geq \sigma_{n}\left(a_{0}\right)$, with at most one exception $f_{0}$ satisfying $\sigma_{M, n+1}\left(f_{0}\right)<\sigma_{n}\left(a_{0}\right)$.

Recently, there is much interest in investigating the fixed points of solutions of differential equations on the plane $\mathbb{C}$ (see [1, 2, 6, 15, 16, 19, 20]). In [4], the fixed points of analytic solutions of differential equations in the unit disc were considered. We obtain some results on the fixed points of analytic solutions of (2).

Theorem 1.4. Let $f$ be a solution of (2), let $a_{j}(j=0,1, \ldots, k-1)$ be $H$-functions and assume that either $F \not \equiv 0$ is an $H$-function or $\sigma_{1}(F)<$ $\sigma_{1}(f)$. If $F(z)-a_{1}(z)-z a_{0}(z) \not \equiv 0$, then $\bar{\lambda}_{1}(f-z)=\lambda_{1}(f)=\bar{\lambda}_{1}(f)=\sigma_{1}(f)$.

TheOREM 1.5. Under the hypothesis of either Theorem 1.2(ii) or Theorem 1.3(iii), if $F(z)-a_{1}(z)-z a_{0}(z) \not \equiv 0$, then all solutions $f$ of (2) satisfy $\bar{\lambda}_{n+1}(f-z)=\lambda_{n+1}(f)=\bar{\lambda}_{n+1}(f)=\sigma_{n+1}(f)$, with at most one exceptional solution.

2. Some lemmas. For the proofs of our main results, we need the lemmas below.

LEMma 2.1 ([10] and [17]). Let $f$ be a meromorphic function in the unit disc $\mathbb{D}$, and let $k \in \mathbb{N}$. Then

$$
m\left(r, f^{(k)} / f\right)=S(r, f),
$$

where

$$
S(r, f)=O\left(\log ^{+} T(r, f)\right)+O\left(\log \frac{1}{1-r}\right),
$$

possibly outside a set $E \subset[0,1)$ with $\int_{E} d r /(1-r)<\infty$. If $f$ is of finite iterated 1-order of growth, then

$$
m\left(r, f^{(k)} / f\right)=O\left(\log \frac{1}{1-r}\right) .
$$

If $f$ is non-admissible, then

$$
m\left(r, f^{\prime} / f\right) \leq \log \frac{1}{1-r}+(2+o(1)) \log \log \frac{1}{1-r} .
$$


The following result was proved in [11]. When $\sigma_{M, n}\left(a_{j}\right)<\sigma_{M, n}\left(a_{0}\right)$ for all $j=1, \ldots, k-1$, the conclusion is also proved in [5].

Lemma 2.2. Let $n \in \mathbb{N}$. If the coefficients $a_{0}(z), \ldots, a_{k-1}(z)$ are analytic in $\mathbb{D}$ such that $\sigma_{M, n}\left(a_{j}\right) \leq \sigma_{M, n}\left(a_{0}\right)$ for all $j=1, \ldots, k-1$, and (3) holds, then all solutions $f \not \equiv 0$ of (1) satisfy $\sigma_{M, n+1}(f)=\sigma_{M, n}\left(a_{0}\right)$.

In [5], one of the present authors and H.-X. Yi also obtained the result below, where the condition $\sigma_{M, n}\left(a_{j}\right)<\sigma_{M, n}\left(a_{0}\right)$ is replaced by $\sigma_{n}\left(a_{j}\right)<$ $\sigma_{n}\left(a_{0}\right)$.

Lemma 2.3. Let $n \in \mathbb{N}$. If the coefficients $a_{0}(z), \ldots, a_{k-1}(z)$ are analytic in $\mathbb{D}$ such that $\sigma_{n}\left(a_{j}\right)<\sigma_{n}\left(a_{0}\right)$ for all $j=1, \ldots, k-1$, then all solutions $f \not \equiv 0$ of (1) satisfy $\alpha_{M} \geq \sigma_{M, n+1}(f) \geq \sigma_{n}\left(a_{0}\right)$, where $\alpha_{M}:=$ $\max \left\{\sigma_{M, n}\left(a_{j}\right): j=0, \ldots, k-1\right\}$.

LEMMA 2.4. Let $\Phi(r)$ be a continuous and positive increasing function, defined for $r$ in $(0,1)$, and set

$$
\sigma_{p}(\Phi)=\limsup _{r \rightarrow 1^{-}} \frac{\log _{p} \Phi(r)}{-\log (1-r)} .
$$

Then for any subset $E$ of $[0,1)$ of finite linear measure, there exists a sequence $\left\{r_{n}\right\}\left(r_{n} \notin E\right)$ such that

$$
\sigma_{p}(\Phi)=\lim _{r_{n} \rightarrow 1^{-}} \frac{\log _{p} \Phi\left(r_{n}\right)}{-\log \left(1-r_{n}\right)} .
$$

Proof. By definition, there exists a sequence $\left\{r_{n}^{\prime}\right\}\left(r_{n}^{\prime} \rightarrow 1^{-}\right)$such that

$$
\lim _{r_{n}^{\prime} \rightarrow 1^{-}} \frac{\log _{p} \Phi\left(r_{n}^{\prime}\right)}{-\log \left(1-r_{n}^{\prime}\right)}=\sigma_{p}(\Phi) .
$$

Set $\int_{E} d r /(1-r)=: \log \delta<\infty$. Since

$$
\int_{r_{n}^{\prime}}^{1-\left(1-r_{n}^{\prime}\right) /(\delta+1)} \frac{d r}{1-r}=\log (\delta+1)
$$

there exists $r_{n} \in\left[r_{n}^{\prime}, 1-\left(1-r_{n}^{\prime}\right) /(\delta+1)\right] \backslash E \subset[0,1)$ such that

$$
\frac{\log _{n}^{+} \Phi\left(r_{n}\right)}{-\log \left(1-r_{n}\right)} \geq \frac{\log _{n}^{+} \Phi\left(r_{n}^{\prime}\right)}{\log \left(\frac{\delta+1}{1-r_{n}^{\prime}}\right)}=\frac{\log _{n}^{+} \Phi\left(r_{n}^{\prime}\right)}{\log (\delta+1)-\log \left(1-r_{n}^{\prime}\right)} \text {. }
$$

Hence

$$
\liminf _{r_{n} \rightarrow 1^{-}} \frac{\log _{n}^{+} \Phi\left(r_{n}\right)}{-\log \left(1-r_{n}\right)} \geq \lim _{r_{n}^{\prime} \rightarrow 1^{-}} \frac{\log _{n}^{+} \Phi\left(r_{n}^{\prime}\right)}{\log (\delta+1)-\log \left(1-r_{n}^{\prime}\right)}=\sigma_{p}(\Phi) .
$$

Therefore

$$
\lim _{r_{n} \rightarrow 1^{-}} \frac{\log _{n}^{+} \Phi\left(r_{n}\right)}{-\log \left(1-r_{n}\right)}=\sigma_{p}(\Phi)
$$


Lemma 2.5. Let $a_{0}, a_{1}, \ldots, a_{k-1}, F \not \equiv 0$ be meromorphic functions in $\mathbb{D}$ and let $f$ be a meromorphic solution of (2) such that $\max \left\{\sigma_{n}(F), \sigma_{n}\left(a_{j}\right)\right.$ : $j=0,1, \ldots, k-1\}<\sigma_{n}(f)$. Then $\bar{\lambda}_{n}(f)=\lambda_{n}(f)=\sigma_{n}(f)$.

Proof. From the equation (2) we see that

$$
\frac{1}{f}=\frac{1}{F}\left(\frac{f^{(k)}}{f}+a_{k-1} \frac{f^{(k-1)}}{f}+\cdots+a_{0}\right) .
$$

If $f$ has a zero at $z_{0} \in \mathbb{D}$ of order $\alpha(>k)$ and $a_{0}, a_{1}, \ldots, a_{k-1}$ are all analytic at $z_{0}$, then from (4) we see that $F$ has a zero at $z_{0}$ of order at least $\alpha-k$. Hence

$$
N(r, 1 / f) \leq k \cdot \bar{N}(r, 1 / f)+N(r, 1 / F) .
$$

It follows from Lemma 2.1 and (4) that

(6) $m(r, 1 / f) \leq m(r, 1 / F)+\sum_{j=0}^{k-1} m\left(r, a_{j}\right)+O\left(\log ^{+} T(r, f)+\log \frac{1}{1-r}\right)$

for all $|z|=r \notin E$, where $E$ is a subset of $[0,1)$ with $\int_{E} d r /(1-r)<\infty$. By (5) and (6), we have

$$
\begin{aligned}
T(r, f)= & T(r, 1 / f)+O(1) \\
\leq & k \bar{N}(r, 1 / f)+T(r, F)+\sum_{j=0}^{k-1} T\left(r, a_{j}\right) \\
& +O\left\{\log \left(\frac{1}{1-r} T(r, f)\right)\right\} \quad(r \notin E) .
\end{aligned}
$$

By Lemma 2.4, there exists a sequence $\left\{r_{n}\right\}\left(r_{n} \notin E\right)$ such that

$$
\lim _{r_{n} \rightarrow 1^{-}} \frac{\log _{n}^{+} T\left(r_{n}, f\right)}{-\log \left(1-r_{n}\right)}=\sigma_{n}(f)=: \sigma .
$$

Set $\max \left\{\sigma_{n}(F), \sigma_{n}\left(a_{j}\right)(j=0, \ldots, k-1)\right\}=: b<\sigma$. Then for any given $\varepsilon$ satisfying $0<2 \varepsilon<\sigma-b$, we have

$$
T\left(r_{n}, f\right) \geq \exp _{n-1}\left(\frac{1}{1-r_{n}}\right)^{\sigma-\varepsilon}, \quad T\left(r_{n}, F\right) \leq \exp _{n-1}\left(\frac{1}{1-r_{n}}\right)^{b+\varepsilon}
$$

and

$$
T\left(r_{n}, a_{j}\right) \leq \exp _{n-1}\left(\frac{1}{1-r_{n}}\right)^{b+\varepsilon}
$$

Hence we get

$$
\max \left\{\frac{T\left(r_{n}, F\right)}{T\left(r_{n}, f\right)}, \frac{T\left(r_{n}, a_{j}\right)}{T\left(r_{n}, f\right)}\right\} \leq \frac{\exp _{n-1}\left(\frac{1}{1-r_{n}}\right)^{b+\varepsilon}}{\exp _{n-1}\left(\frac{1}{1-r_{n}}\right)^{\sigma-\varepsilon}} \rightarrow 0 \quad\left(r_{n} \rightarrow 1^{-}\right) .
$$


Hence for $r_{n} \rightarrow 1^{-}$, we obtain

$$
\begin{aligned}
T\left(r_{n}, F\right) & \leq \frac{1}{k+3} T\left(r_{n}, f\right), \\
T\left(r_{n}, a_{j}\right) & \leq \frac{1}{k+3} T\left(r_{n}, f\right) \quad(j=0, \ldots, k-1) .
\end{aligned}
$$

Furthermore, since $\sigma_{n}(f)>0$, by Lemma 2.1 we get

$$
O\left\{\log \left(\frac{1}{1-r_{n}} T\left(r_{n}, f\right)\right)\right\}=o\left(T\left(r_{n}, f\right)\right) .
$$

Thus for $r_{n} \rightarrow 1^{-}$, we have

$$
O\left\{\log \left(\frac{1}{1-r_{n}} T\left(r_{n}, f\right)\right)\right\} \leq \frac{1}{k+3} T\left(r_{n}, f\right) .
$$

Now we deduce from (7)-(9) that $T\left(r_{n}, f\right) \leq k(k+3) \bar{N}\left(r_{n}, 1 / f\right)$. This gives $\bar{\lambda}_{n}(f)=\lambda_{n}(f)=\sigma_{n}(f)$.

\section{Proofs.}

Proof of Theorem 1.1. From the equation (2), we see that $\sigma_{1}(f) \geq \sigma_{1}(F)$. Let $f_{1}, \ldots, f_{k}$ be a solution base of (1). Then by the elementary theory of differential equations (see, e.g., [14]), any solution of (2) can be represented in the form

$$
f=\left(A_{1}+C_{1}\right) f_{1}+\left(A_{2}+C_{2}\right) f_{2}+\cdots+\left(A_{k}+C_{k}\right) f_{k},
$$

where $C_{1}, \ldots, C_{k} \in \mathbb{C}$ and $A_{1}, \ldots, A_{k}$ are analytic in $\mathbb{D}$ given by the system of equations

$$
\left\{\begin{array}{l}
A_{1}^{\prime} f_{1}+A_{2}^{\prime} f_{2}+\cdots+A_{k}^{\prime} f_{k}=0, \\
A_{1}^{\prime} f_{1}^{\prime}+A_{2}^{\prime} f_{2}^{\prime}+\cdots+A_{k}^{\prime} f_{k}^{\prime}=0, \\
\cdots \\
A_{1}^{\prime} f_{1}^{(k-2)}+A_{2}^{\prime} f_{2}^{(k-2)}+\cdots+A_{k}^{\prime} f_{k}^{(k-2)}=0, \\
A_{1}^{\prime} f_{1}^{(k-1)}+A_{2}^{\prime} f_{2}^{(k-1)}+\cdots+A_{k}^{\prime} f_{k}^{(k-1)}=F .
\end{array}\right.
$$

Since the Wronskian of $f_{1}, \ldots, f_{k}$ satisfies $W\left(f_{1}, \ldots, f_{k}\right)=\exp \left(-\int a_{k-1} d z\right)$, we obtain

$$
A_{j}^{\prime}=F \cdot G_{j}\left(f_{1}, \ldots, f_{k}\right) \cdot \exp \left(\int a_{k-1} d z\right) \quad(j=1, \ldots, k),
$$

where $G_{j}\left(f_{1}, \ldots, f_{k}\right)$ is a differential polynomial of $f_{1}, \ldots, f_{k}$ and of their derivatives, with constant coefficients. From Theorem 1 in [13], all solutions $f_{1}, \ldots, f_{k}$ of the homogeneous differential equation (1) satisfy

$$
\sigma_{1}\left(f_{i}\right) \leq \sigma_{M, 1}\left(f_{i}\right) \leq \gamma:=\max \left\{0, \max _{j=0, \ldots, k-1} \frac{D_{M}\left(a_{j}\right)}{k-j}-1\right\}
$$


for $i=1, \ldots, k$. For an analytic function $g$ in $\mathbb{D}$, we get $\sigma_{1}(g)=\sigma_{1}\left(g^{\prime}\right)$ (see [18, Theorem V.28]), $\sigma_{M, 1}(g)=\sigma_{M, 1}\left(g^{\prime}\right)$ (see [5, Proposition 1.2(ii)]), and so inductively, $\sigma_{1}(g)=\sigma_{1}\left(g^{(n)}\right)$ and $\sigma_{M, 1}(g)=\sigma_{M, 1}\left(g^{(n)}\right)$ for any $n \in \mathbb{N}$. By Proposition 2(ii) in [7], we get $D_{M}\left(\int a_{k-1} d z\right)=D_{M}\left(a_{k-1}\right)-1 \leq \gamma$. Thus $\sigma_{M, 1}\left(\exp \left(\int a_{k-1} d z\right) t\right) \leq \gamma$. Therefore, all solutions $f$ of the non-homogeneous differential equation (2) satisfy

$$
\sigma_{1}(F) \leq \sigma_{1}(f) \leq \sigma_{M, 1}(f) \leq \gamma:=\max \left\{0, \max _{j=0, \ldots, k-1} \frac{D_{M}\left(a_{j}\right)}{k-j}-1\right\} .
$$

If $\sigma_{1}(f)>\sigma_{1}(F)$, then we see immediately from Lemma 2.5 that $\bar{\lambda}_{1}(f)=$ $\lambda_{1}(f)=\sigma_{1}(f)$. Next we assume that $F$ is an $H$-function. Since all solutions of (2) are of finite order by the above growth estimate of solutions, we can immediately conclude from Lemma 2.1 and (4) that

$$
\begin{aligned}
m(r, 1 / f) \leq m(r, 1 / F)+m(r, F / f) & \leq \sum_{i=0}^{k-1} m\left(r, a_{i}\right)+\sum_{j=1}^{k} m\left(r, f^{(j)} / f\right) \\
& =O\left(\log \frac{1}{1-r}\right) .
\end{aligned}
$$

Hence we get $\lambda_{1}(f)=\sigma_{1}(f)$. Moreover, we see from (2) that any solution $f$ has at most finitely many zeros of multiplicity $\geq k+1$ in $\mathbb{D}$, and that if $f$ has a zero at $z_{0} \in \mathbb{D}$ of order $\alpha(>k)$, then $F$ has a zero at $z_{0}$ of order at least $\alpha-k$. Hence

$$
N(r, 1 / f) \leq k \cdot \bar{N}(r, 1 / f)+N(r, 1 / F)=k \cdot \bar{N}(r, 1 / f)+O\left(\log \frac{1}{1-r}\right),
$$

and so $\bar{\lambda}_{1}(f)=\lambda_{1}(f)$. Therefore $\bar{\lambda}_{1}(f)=\lambda_{1}(f)=\sigma_{1}(f)$.

Proof of Theorem 1.2. Assume that $\left\{f_{1}, \ldots, f_{k}\right\}$ is a solution base of (1). By Lemma 2.2, we know that $\sigma_{M, n+1}\left(f_{j}\right)=\sigma_{M, n}\left(a_{0}\right)(j=1, \ldots, k)$. Thus we also get the equations $10-12$, and hence

$$
\sigma_{M, n+1}(f) \leq \max \left\{\sigma_{M, n+1}(F), \sigma_{M, n}\left(a_{0}\right)\right\} .
$$

(i) If $\sigma_{M, n+1}(F)>\sigma_{M, n}\left(a_{0}\right)$, it follows from (13) and (2) that $\sigma_{M, n+1}(f)$ $=\sigma_{M, n+1}(F)$.

(ii) If $\sigma_{M, n+1}(F)<\sigma_{M, n}\left(a_{0}\right)$, it follows immediately from (13) that all solutions $f(z)$ of (2) satisfy $\sigma_{M, n+1}(f) \leq \sigma_{M, n}\left(a_{0}\right)$.

Now we assert that all solutions $f$ of (2) satisfy $\sigma_{M, n+1}(f)=\sigma_{M, n}\left(a_{0}\right)$ with at most one exception. In fact, if there exist two distinct solutions $g_{1}, g_{2}$ of (2) that satisfy $\sigma_{M, n+1}\left(g_{i}\right)<\sigma_{M, n}\left(a_{0}\right)(i=1,2)$, then $g=g_{1}-g_{2}$ satisfies $\sigma_{M, n+1}(g)=\sigma_{M, n+1}\left(g_{1}-g_{2}\right)<\sigma_{M, n}\left(a_{0}\right)$. But $g=g_{1}-g_{2}$ is a nonzero solution of (1) satisfying $\sigma_{M, n+1}(g)=\sigma_{M, n+1}\left(g_{1}-g_{2}\right)=\sigma_{M, n}\left(a_{0}\right)$ by Lemma 2.2. This is a contradiction. 
By Lemma 2.5, we infer that all solutions $f$ of $(2)$ with $\sigma_{M, n+1}(f)=$ $\sigma_{M, n}\left(a_{0}\right)$ satisfy $\sigma_{M, n+1}(f)=\bar{\lambda}_{n+1}(f)=\lambda_{n+1}(f)$.

Proof of Theorem 1.3. Assume that $\left\{f_{1}, \ldots, f_{k}\right\}$ is a solution base of (1). Then by Lemma 2.3 . we know that $\alpha_{M} \geq \sigma_{M, n+1}\left(f_{j}\right) \geq \sigma_{n}\left(a_{0}\right)$. Thus we also get the equations $(10)-(12)$, and hence

$$
\sigma_{M, n+1}(f) \leq \max \left\{\sigma_{M, n+1}(F), \alpha_{M}\right\} .
$$

(i) If $\sigma_{M, n+1}(F)>\alpha_{M}$, it follows from (14) and (2) that $\sigma_{M, n+1}(f)=$ $\sigma_{M, n+1}(F)$.

(ii) If $\sigma_{M, n+1}(F)<\alpha_{M}$, it follows from (14) and (2) that all solutions $f(z)$ of (2) satisfy $\sigma_{M, n+1}(f) \leq \alpha_{M}$.

Now we assert that all solutions $f$ of (2) satisfy $\sigma_{M, n+1}(f) \geq \sigma_{n}\left(a_{0}\right)$ with at most one exception. In fact, if there exist two distinct solutions $g_{1}, g_{2}$ of 2 with $\sigma_{M, n+1}\left(g_{i}\right)<\sigma_{n}\left(a_{0}\right)(i=1,2)$, then $g=g_{1}-g_{2}$ satisfies $\sigma_{M, n+1}(g)=\sigma_{M, n+1}\left(g_{1}-g_{2}\right)<\sigma_{n}\left(a_{0}\right)$. But $g$ is a nonzero solution of (1) satisfying $\sigma_{M, n+1}(g)=\sigma_{M, n+1}\left(g_{1}-g_{2}\right) \geq \sigma_{n}\left(a_{0}\right)$ by Lemma 2.3 . This is a contradiction.

(iii) If $\sigma_{M, n+1}(F)<\sigma_{n}\left(a_{0}\right) \leq \alpha_{M}$, then by (ii) and Lemma 2.5, all solutions $f$ of $(2)$ satisfy $\alpha_{M} \geq \sigma_{M, n+1}(f)=\bar{\lambda}_{n+1}(f)=\lambda_{n+1}(f) \geq \sigma_{n}\left(a_{0}\right)$, with at most one exception $f_{0}$ satisfying $\sigma_{M, n+1}\left(f_{0}\right)<\sigma_{n}\left(a_{0}\right)$.

Proof of Theorem 1.4. Set $g(z)=f(z)-z, z \in \mathbb{D}$. It is obvious that $\bar{\lambda}_{1}(f-z)=\bar{\lambda}_{1}(g)$ and $\sigma_{1}(f)=\sigma_{1}(g)$. The equation (2) becomes

$$
g^{(k)}+a_{k-1}(z) g^{(k-1)}+\cdots+a_{0}(z) g=F(z)-\left(a_{1}(z)+z a_{0}(z)\right) .
$$

Assume that $F(z)-\left(a_{1}(z)+z a_{0}(z)\right) \not \equiv 0$. Then by Theorem 1.1 we have $\sigma_{1}(g)=\bar{\lambda}_{1}(f)=\sigma_{1}(f)>\max \left\{\sigma_{1}\left(a_{j}\right), \sigma_{1}\left(F-a_{1}-z a_{0}\right): j=0,1, \ldots, k-1\right\}$. Hence, we deduce by Lemma 2.5 that $\bar{\lambda}_{1}(g)=\sigma_{1}(g)$. Therefore, we obtain $\bar{\lambda}_{1}(f-z)=\bar{\lambda}_{1}(g)=\sigma_{1}(g)=\bar{\lambda}_{1}(f)=\sigma_{1}(f)$.

Proof of Theorem 1.5. Set $g(z)=f(z)-z, z \in \mathbb{D}$. It is obvious that $\bar{\lambda}_{n+1}(f-z)=\bar{\lambda}_{n+1}(g)$ and $\sigma_{n+1}(f)=\sigma_{n+1}(g)$. The equation (2) becomes

$$
g^{(k)}+A_{k-1}(z) g^{(k-1)}+\cdots+a_{0}(z) g=F(z)-\left(a_{1}(z)+z a_{0}(z)\right) .
$$

Assume that $F(z)-\left(a_{1}(z)+z a_{0}(z)\right) \not \equiv 0$. Then by Theorem 1.2(ii) or Theorem 1.3(iii), with at most one exceptional solution, all solutions $f$ of (2) satisfy $\sigma_{n+1}(f)=\bar{\lambda}_{n+1}(f)$. Now we may assume that $f$ is not the exceptional solution, and thus for all $j=0,1, \ldots, k-1$, we have

$$
\sigma_{n+1}(g)=\bar{\lambda}_{n+1}(f)=\sigma_{n+1}(f)>\max \left\{\sigma_{n+1}\left(a_{j}\right), \sigma_{n+1}\left(F-a_{1}-z a_{0}\right)\right\} .
$$

Hence, we deduce by Lemma 2.5 that $\bar{\lambda}_{n+1}(g)=\sigma_{n+1}(g)$. Therefore, we obtain $\bar{\lambda}_{n+1}(f-z)=\bar{\lambda}_{n+1}(g)=\sigma_{n+1}(g)=\bar{\lambda}_{n+1}(f)=\sigma_{n+1}(f)$, with at most one exceptional solution. 
Acknowledgements. The authors would like to thank the referee(s) for making valuable suggestions and comments.

This work was supported by the NNSF of China (No. 10771121) and the NSF of Jiangxi of China (No. 2008GQS0075).

\section{References}

[1] B. Belaïdi, On the iterated order and the fixed points of entire solutions of some complex linear differential equations, Electron. J. Qual. Theory Differential Equations 2006, no. 9, 11 pp.

[2] - Oscillation of fixed points of solutions of some linear differential equations, Acta Math. Univ. Comenian. 77 (2008), 263-269.

[3] D. Benbourenane and L. R. Sons, On global solutions of complex differential equations in the unit disc, Complex Var. Theory Appl. 49 (2004), 913-925.

[4] T.-B. Cao, The growth, oscillation and fixed points of solutions of complex linear differential equations in the unit disc, J. Math. Anal. Appl. 352 (2009), 739-748.

[5] T.-B. Cao and H.-X. Yi, The growth of solutions of linear differential equations with coefficients of iterated order in the unit disc, J. Math. Anal. Appl. 319 (2006), $278-294$.

[6] Z.-X. Chen, The fixed points and hyper-order of solutions of second order complex differential equations, Acta Math. Sci. Ser. A Chin. Ed. 20 (2000), 425-432 (in Chinese).

[7] Z.-X. Chen and K.-H. Shon, The growth of solutions of differential equations with coefficients of small growth in the disc, J. Math. Anal. Appl. 297 (2004), 285-304.

[8] I. Chyzhykov, G. Gundersen and J. Heittokangas, Linear differential equations and logarithmic derivative estimates, Proc. London Math. Soc. 86 (2003), 735-754.

[9] W. Hayman, Meromorphic Functions, Clarendon Press, Oxford, 1964.

[10] J. Heittokangas, On complex differential equations in the unit disc, Ann. Acad. Sci. Fenn. Math. Diss. 122 (2000), 54 pp.

[11] J. Heittokangas, R. Korhonen and J. Rättyä, Fast growing solutions of linear differential equations in the unit disc, Results Math. 49 (2006), 265-278.

[12] - - - - Linear differential equations with coefficients in weighted Bergman and Hardy spaces, Trans. Amer. Math. Soc. 360 (2007), 1035-1055.

[13] R. Korhonen and J. Rättyä, Linear differential equations in the unit disc with analytic solutions of finite order, Proc. Amer. Math. Soc. 135 (2007), 1355-1363.

[14] I. Laine, Nevanlinna Theory and Complex Differential Equations, de Gruyter, Berlin, 1993.

[15] I. Laine and J. Rieppo, Differential polynomials generated by linear differential equations, Complex Var. Theory Appl. 49 (2004), 897-911.

[16] M.-S. Liu and X.-M. Zhang, Fixed points of meromorphic solutions of higher order linear differential equations, Ann. Acad. Sci. Fenn. Math. 31 (2006), 191-211.

[17] D. Shea and L. Sons, Value distibution theory for meromorphic functions of slow growth in the disk, Houston J. Math. 12 (1986), 249-266.

[18] M. Tsuji, Potential Theory in Modern Function Theory, Chelsea, New York, 1975, reprint of the 1959 edition.

[19] J. Wang and W.-R. Lü, The fixed points and hyper-order of solutions of second order linear differential equations with meromorphic coeffcients, Acta Math. Appl. Sin. 27 (2004), 72-80 (in Chinese). 
[20] J. Wang and H.-X. Yi, Fixed points and hyper order of differential polynomials generated by solutions of differential equation, Complex Var. Theory Appl. 48 (2003), 83-94.

[21] L. Yang, Value Distribution Theory, Springer, Berlin, 1993, and Science Press, Beijing, 1982.

Ting-Bin Cao

Department of Mathematics

Nanchang University

Nanchang 330031, China

E-mail: tbcao@ncu.edu.cn
Zhong-Shu Deng (corresponding author)

Editorial office of Journal

Nanchang University

Nanchang 330047, China

E-mail: dengzhongshu@ncu.edu.cn

Received 13.1.2009

and in final form 12.10.2009 
